\title{
Computed tomography of the thorax of calves from birth to the age of 105 days
}

Ohlerth, Stefanie ; Augsburger, Heinz ; Abé, Maximilian ; Hatz, L ; Braun, Ueli ; Ringer, Simone K

\begin{abstract}
The present study was undertaken to provide computed tomographic (CT) reference values for structures in the thorax of the calf. Six clinically healthy Holstein-Friesian calves were anaesthetized. Transverse pre- and postcontrast images with a reconstructed $1.5-\mathrm{mm}$ slice thickness were obtained using a multislice-CT scanner at 6 different time points from birth to 105 days of age. Absolute and relative measurements of the trachea, heart, cranial and caudal vena cava, thoracic aorta, right and left principal bronchi, right and left caudal lobar bronchi and the accompanying branches of the right and left pulmonary artery and vein, thoracic lymph nodes and lung density were taken for every time point. All animals were euthanized after the last CT scan, and 4 calves were frozen to generate an atlas comparing gross anatomy with CT. During the study, 4 animals temporarily showed coughing and mucopurulent nasal discharge, and mild to moderate bronchopneumonia and pleuritis were diagnosed using CT. Animals recovered with treatment; however, mild to moderate CT changes remained throughout the study. Even in the 2 clinically normal animals, mild bronchopneumonia was diagnosed on CT.
\end{abstract}

DOI: https://doi.org/10.1024/0036-7281/a000636

Posted at the Zurich Open Repository and Archive, University of Zurich

ZORA URL: https://doi.org/10.5167/uzh-99845

Journal Article

Supplemental Material

Originally published at:

Ohlerth, Stefanie; Augsburger, Heinz; Abé, Maximilian; Hatz, L; Braun, Ueli; Ringer, Simone K (2014). Computed tomography of the thorax of calves from birth to the age of 105 days. Schweizer Archiv für Tierheilkunde, 156(10):489-497.

DOI: https://doi.org/10.1024/0036-7281/a000636 
Table 1: Descriptive statistics of thoracic measurements in 6 clinically normal calves of 6 consecutive CT examinations 3 weeks apart [mean, standard deviation (minimum-maximum)]; CSA: cross-sectional area; *first measurement which was significantly different from first CT scan: $p<0.05$ )

\begin{tabular}{|c|c|c|c|c|c|c|}
\hline \multirow[t]{2}{*}{ Variable } & \multicolumn{6}{|c|}{ CT scan } \\
\hline & Scan 1 (1-2 days old) & Scan 2 (21 days old) & Scan 3 (42 days old) & Scan 4 (63 days old) & Scan 5 (84 days old) & Scan 6 (105 days old) \\
\hline Thoracic height at $\mathrm{T} 1(\mathrm{~cm})$ & $11.5,0.78(10.5-12.6)$ & $\begin{array}{llll}12.2, & 0.47 & (11.5 & - \\
12.8) & & & \\
\end{array}$ & $\begin{array}{llll}13.2, & 0.87 & (11.9 & - \\
13.9)^{*} & & & \\
\end{array}$ & $13.9,1.0(12.5-15.5)$ & $14.5,1.2(12.5-15.8)$ & $15.8,1.6(13.4-18.4)$ \\
\hline Thoracic height at $\mathrm{T} 4(\mathrm{~cm})$ & $14.8,1.53(12.7-16.9)$ & $\begin{array}{llll}16.9, & 0.89 & (15.3 \quad- \\
17.8)^{*} & & & \end{array}$ & $\begin{array}{llll}19.1, & 1.36 & (16.8 & - \\
20.4) & & & \\
\end{array}$ & $\begin{array}{llll}19.5, & 0.79 & (19.1 & - \\
21.3) & & & \\
\end{array}$ & $20.7,1.33(18.3-21.9)$ & $21.7,1.07(19.9-22.7)$ \\
\hline Thoracic height at $\mathrm{T} 8(\mathrm{~cm})$ & $16.0,1.33(14.1-17.5)$ & $\begin{array}{llll}18.2, & 1.06 \quad(17.2 \quad- \\
19.7)^{*} & & & \end{array}$ & $20.5,0.6(19.6-21.1)$ & $\begin{array}{llll}21.2, & 0.89 & (20.2 & - \\
22.0) & & & \end{array}$ & $23.1,0.66(22.7-23.6)$ & $23.4,0.32(23.2-23.7)$ \\
\hline Thoracic width at $\mathrm{T} 1(\mathrm{~cm})$ & $6.0,0.44(5.3-6.4)$ & $6.1,0.43(5.2-6.4)$ & $6.7,0.43(5.9-7.0)^{*}$ & $7.1,0.47(6.3-7.5)$ & $7.3,0.53(6.4-7.8)$ & $7.4,1.04(6.2-9.4)$ \\
\hline Thoracic width at $\mathrm{T} 4(\mathrm{~cm})$ & $9.9,0.65(9.2-10.7)$ & $\begin{array}{llll}11.1, & 0.38 & (10.5 \quad- \\
11.6)^{*} & & & \end{array}$ & $\begin{array}{llll}12.9, & 2.06 & (10.7 & - \\
16.3) & & & \\
\end{array}$ & $\begin{array}{llll}13.6, & 1.28 & (12.0 & - \\
15.0) & & & \end{array}$ & $15.6,2.84(12.3-18.9)$ & $18.4,3.8(13.8-22.2)$ \\
\hline Thoracic width at $\mathrm{T} 8(\mathrm{~cm})$ & $12.9,1.02(11.3-14.2)$ & $\begin{array}{llll}15.0, & 0.77 & (14.1 & - \\
15.9)^{*} & & & \end{array}$ & $\begin{array}{llll}17.9, & 1.41 & (16.1 & - \\
19.8) & & & \\
\end{array}$ & $\begin{array}{llll}20.8, & 2.65 & (18.3 & - \\
25.3) & & & \end{array}$ & $27.2,6.21(19.6-36.9)$ & $29.7,5.21(24.6-35.9)$ \\
\hline Cardiac width $(\mathrm{cm})$ & $9.7,0.83(9.1-11.2)$ & $10.9,0.74(9.6-11.8)^{*}$ & $11.8,0.37(11.3-12.3)$ & $12.2,0.62(11.2-12.9)$ & $12.0,1.21(10.0-13.6)$ & $12.6,0.44(12.2-13.4)$ \\
\hline Cardiac height $(\mathrm{cm})$ & $14.4,0.91(13.6-16.1)$ & $15.1,0.67(13.9-16.0)$ & $15.3,0.59(14.2-15.9)$ & $\begin{array}{lll}16.7, & 1.65 & (13.9- \\
18.9)^{*} & & \\
\end{array}$ & $15.9,1.16(14.4-17.3)$ & $17.2,0.58(16.1-17.7)$ \\
\hline Vertebral heart score (VHS) & $9.3,0.3(9.0-10.0)$ & $9.3,0.24(9.0-9.5)$ & $9.1,0.21(9.0-9.5)$ & $8.8,0.22(8.5-9.0)^{*}$ & $7.9,0.49(7.5-9.0)$ & $8.2,0.27(8.0-9.0)$ \\
\hline Maximal height of aorta $(\mathrm{cm})$ & $1.7,0.21(1.4-2.0)$ & $2.0,0.19(1.7-2.2)^{*}$ & $2.1,0.1(2.0-2.3)$ & $2.4,0.22(2.0-2.6)$ & $2.6,0.26(2.2-2.9)$ & $2.7,0.31(2.4-3.2)$ \\
\hline $\begin{array}{l}\text { Maximal height of cranial vena cava } \\
(\mathrm{cm})\end{array}$ & $1.7,0.21(1.75-2.0)$ & $1.9,0.24(1.5-2.2)$ & $2.1,0.21(1.7-2.3)^{*}$ & $2.2,0.22(1.0-2.5)$ & $2.3,0.34(1.7-2.6)$ & $2.3,0.28(1.8-2.6)$ \\
\hline $\begin{array}{l}\text { Maximal height of caudal vena cava } \\
(\mathrm{cm})\end{array}$ & $2.0,0.27(1.7-2.5)$ & $1.8,0.42(1.4-2.4)$ & $1.8,0.12(1.6-2.0)$ & $2.1,0.2(1.2-2.5)$ & $1.8,0.27(1.4-2.1)$ & $2.0,0.25(1.7-2.4)$ \\
\hline Maximal height of caudal vena & $1.2,0.14(1.0-1.4)$ & $0.9,0.18(0.7-1.2)^{*}$ & $0.8,0.07(0.7-0.9)$ & $0.9,0.24(0.5-1.2)$ & $0.7,0.08(0.6-0.8)$ & $0.8,0.15(0.5-0.9)$ \\
\hline
\end{tabular}




\begin{tabular}{|c|c|c|c|c|c|c|}
\hline cava/aorta & & & & & & \\
\hline $\begin{array}{l}\text { Maximal height of caudal vena } \\
\text { cava/length of vertebra at the level of } \\
\text { the bifurcation of the trachea }\end{array}$ & $0.8,0.11(0.7-1.0)$ & $0.7,0.15(0.5-0.9)^{*}$ & $0.6,0.06(0.6-0.7)$ & $0.6,0.14(0.4-0.8)$ & $0.5,0.09(0.4-0.6)$ & $0.6,0.09(0.5-0.7)$ \\
\hline Tracheal height at thoracic inlet $(\mathrm{cm})$ & $2.0,0.22(1.8-2.4)$ & $2.3,0.32(1.9-2.7)$ & $2.3,0.27(1.9-2.6)$ & $2.3,0.32(1.9-2.8)$ & $2.3,0.33(1.8-2.7)$ & $2.7,0.33(2.3-3.3)^{*}$ \\
\hline Tracheal width at thoracic inlet $(\mathrm{cm})$ & $2.0,0.19(1.7-2.2)$ & $2.0,0.21(1.8-2.4)$ & $2.1,0.08(2.0-2.2)$ & $2.1,0.25(1.6-2.3)$ & $2.0,0.17(1.8-2.3)$ & $2.4,0.26(2.1-2.7)^{*}$ \\
\hline CSA of trachea at thoracic inlet $\left(\mathrm{cm}^{2}\right)$ & $3.3,0.48(2.7-4.2)$ & $3.9,0.81(3.1-5.4)$ & $4.0,0.79(2.8-5.0)$ & $4.0,0.99(2.6-5.3)$ & $3.7,1.28(2.4-5.4)$ & $5.3,1.3(3.6-7.5)^{*}$ \\
\hline CSA of trachea at bifurcation $\left(\mathrm{cm}^{2}\right)$ & $3.1,0.79(2.1-4.4)$ & $3.7,0.92(2.4-5.2)$ & $3.8,0.62(3.1-4.6)$ & $4.0,0.59(3.4-5.0)^{*}$ & $4.2,0.82(3.5-5.7)$ & $5.3,1.5(4.0-8.0)$ \\
\hline $\begin{array}{l}\text { Tracheal height/thoracic height at } \\
\text { thoracic inlet }\end{array}$ & $0.18,0.01(0.17-0.19)$ & $0.19,0.02(0.17-0.22)$ & $0.18,0.02(0.13-0.19)$ & $0.16,0.02(0.12-0.19)$ & $0.16,0.02(0.12-0.18)$ & $0.17,0.02(0.14-0.19)$ \\
\hline $\begin{array}{l}\text { Outer CSA of right principal } \\
\text { bronchus }\left(\mathrm{cm}^{2}\right)\end{array}$ & $1.8,0.4(1.4-2.3)$ & $2.6,0.5(2.0-3.5)^{*}$ & $2.9,0.42(2.9-4.2)$ & $3.7,0.71(2.7-4.6)$ & $4.0,0.49(3.2-4.5)$ & $4.3,0.89(3.5-6.0)$ \\
\hline $\begin{array}{l}\text { Inner CSA of right principal } \\
\text { bronchus }\left(\mathrm{cm}^{2}\right)\end{array}$ & $1.2,0.29(0.8-1.5)$ & $1.5,0.38(1.2-2.2)$ & $2.0,0.41(1.6-2.7)^{*}$ & $1.9,0.45(1.2-2.4)$ & $2.2,0.32(1.6-2.6)$ & $2.3,0.72(1.6-3.7)$ \\
\hline $\begin{array}{l}\text { CSA of wall of right principal } \\
\text { bronchus }\left(\mathrm{cm}^{2}\right)\end{array}$ & $0.6,0.11(0.5-0.8)$ & $1.1,0.26(0.7-1.5)^{*}$ & $1.4,0.09(1.3-1.5)$ & $1.7,0.3(1.3-2.2)$ & $1.8,0.29(1.5-2.3)$ & $2.0,0.37(1.5-2.4)$ \\
\hline $\begin{array}{l}\text { Outer CSA of left principal bronchus } \\
\left(\mathrm{cm}^{2}\right)\end{array}$ & $1.7,0.39(1.3-2.3)$ & $2.4,0.7(1.9-3.8)$ & $3.3,0.8(2.3-4.3)^{*}$ & $3.1,0.72(2.2-4.3)$ & $3.9,0.69(3.0-4.6)$ & $4.0,1.16(2.6-5.7)$ \\
\hline $\begin{array}{l}\text { Inner CSA of left principal bronchus } \\
\left(\mathrm{cm}^{2}\right)\end{array}$ & $1.1,0.26(0.81-1.4)$ & $1.5,0.5(1.0-2.7)$ & $1.9,0.5(1.3-2.7)^{*}$ & $1.7,0.43(1.0-2.2)$ & $2.2,0.5(1.1-2.6)$ & $2.2,0.68(1.6-3.8)$ \\
\hline $\begin{array}{l}\text { CSA of wall of left principal } \\
\text { bronchus }\left(\mathrm{cm}^{2}\right)\end{array}$ & $0.6,0.16(0.4-0.9)$ & $0.8,0,18(0.6-1.1)^{*}$ & $1.3,0.36(0.9-1.8)$ & $1.4,0.3(1.2-2.1)$ & $1.9,0.35(1.3-2.1)$ & $1.7,0.49(0.94-2.2)$ \\
\hline Interbronchial angle $\left(^{\circ}\right)$ & $41.1,3.6(36.1-46.5)$ & $42.0,4.7(35.2-47.4)$ & $47.7,7.9(37.8-61.1)$ & $49.2,7.0(38.3-58.6)^{*}$ & $58.0,12.6(41.2-75.6)$ & $63.7,8.7(51.2-73.3)$ \\
\hline $\begin{array}{l}\text { Inner CSA of left caudal lobar } \\
\text { bronchus }\left(\mathrm{cm}^{2}\right)\end{array}$ & $0.6,0.14(0.4-0.8)$ & $0.3,0.09(0.2-0.4)^{*}$ & $0.4,0.17(0.1-0.6)$ & $0.4,0.09(0.3-0.5)$ & $0.4,0.16(0.2-0.6)$ & $0.5,0.15(0.3-0.8)$ \\
\hline CSA of caudal lobar branch of left & $0.5,0.13(0.4-0.8)$ & $0.3,0.04(0.2-0.4)^{*}$ & $0.4,0.16(0.2-0.7)$ & $0.6,0.19(0.4-0.9)$ & $0.5,0.23(0.3-0.8)$ & $0.6,0.12(0.4-0.8)$ \\
\hline
\end{tabular}




\begin{tabular}{|l|l|l|l|l|l|l|}
\hline pulmonary artery $\left(\mathrm{cm}^{2}\right)$ & & & & & \\
\hline $\begin{array}{l}\text { CSA of caudal lobar branch of left } \\
\text { pulmonary vein }\left(\mathrm{cm}^{2}\right)\end{array}$ & $0.6,0.21(0.3-1.0)$ & $0.3,0.06(0.2-0.4)^{*}$ & $0.5,0.15(0.2-0.7)$ & $0.5,0.15(0.3-0.7)$ & $0.5,0.28(0.2-0.9)$ & $0.6,0.15(0.4-0.7)$ \\
\hline $\begin{array}{l}\text { Inner CSA of right caudal lobar } \\
\text { bronchus }\left(\mathrm{cm}^{2}\right)\end{array}$ & $0.7,0.14(0.5-0.8)$ & $0.3,0.14(0.2-0.5)^{*}$ & $0.4,0.2(0.2-0.7)$ & $0.4,0.11(0.2-0.5)$ & $0.5,0.16(0.2-0.7)$ & $0.5,0.2(0.3-0.8)$ \\
\hline $\begin{array}{l}\text { CSA of caudal lobar branch of right } \\
\text { pulmonary artery }\left(\mathrm{cm}^{2}\right)\end{array}$ & $0.7,0.23(0.5-1.1)$ & $0.4,0.26(0.2-0.9)^{*}$ & $0.5,0.21(0.3-0.8)$ & $0.5,0.13(0.4-0.7)$ & $0.5,0.13(0.4-0.7)$ & $0.7,0.16(0.4-0.8)$ \\
\hline $\begin{array}{l}\text { CSA of caudal lobar branch of right } \\
\text { pulmonary vein }\left(\mathrm{cm}^{2}\right)\end{array}$ & $0.7,0.13(0.5-0.9)$ & $0.5,0.4(0.2-1.4)^{*}$ & $0.5,0.22(0.3-0.7)$ & $0.5,0.2(0.3-0.7)$ & $0.5,0.21(0.3-0.9)$ & $0.7,0.3(0.3-1.0)$ \\
\hline
\end{tabular}

